Editorial

\title{
Management of colorectal hepatic metastases: time for targeted patient care
}

From the slightly less than 150,000 new cases of colorectal cancer (CRC) in the US every year, approximately $15 \%$ will have hepatic metastatic disease at the time of diagnosis, whereas another $50 \%$ will develop them during the course of their disease. ${ }^{1}$ Although recent advances in neoadjuvant and adjuvant therapy after colon resection have decreased the number of cases with metastatic disease and newer chemotherapeutic agents, such as irinocetan, cetuximab and bevacizumab have improved the response rates, the two-year survival with only chemotherapy remains at $40 \%$ at best for patients with metastatic disease. The result is that surgical treatments remain the main therapeutic approach as resection of the hepatic metastases with a curative intent has been shown to lead to 5-year survivals of 30$40 \%{ }^{2}$ Adding to that, a combination of improved surgical techniques and better pre- and intra-operative patient assessment have led to a significant increase in the number and type of patients whose hepatic metastatic disease is now considered resectable, as opposed to few years ago, when these same patients would not have enjoyed the possibility of a curative treatment. The critical factors have been-

i. New surgical and interventional techniques (such as radiofrequency ablation, chemoembolization, microwave ablation among others) that can be used in combination.

ii. Improved ability to assess and ameliorate the liver remnant after a hepatectomy.

iii. Intraoperative radiologic and navigation systems, that allows a much more focused approach, where more hepatic parenchyma is spared.

iv. The increased ability of experienced anesthesia teams to successfully perioperatively manages these challenging patients.

Despite this progress, one of the things that remains apparent when we see different patients with similar disease load responding to the same treatment in different manners is that patient and tumor biology still dictate the ultimate result (as evidenced by survival and recurrence).

Components of the stroma include angiogenic cells, fibroblasts and cells of the immune system. These cells of the microenvironment through processes such as growth suppressor evasion, proliferative signaling and resistance to apoptosis and cell death are directly involved in the carcinogenic process, including the aspects of metastatic potential and chemo resistance. The balance of the relation between the tumor microenvironment and the tumor itself can lead to tumor progression through an active network of nontumoral cells and molecules that create a permissive environment for the establishment and progression of hepatic metastatic disease. ${ }^{3}$ The other way that the hepatic microenvironment can play a role is through the existence of liver disease, such as hepatitis B or C viral infections, which activate the immune system and through the eventual establishment of cirrhosis lead to oncogenic transformation of the virally infected hepatocytes.

In addition to the liver and tumor microenvironment, there is the element of genetic involvement. Through powerful genome-wide
Volume I Issue I - 2014

Georgios Tsoulfas

Department of Surgery, Aristotle University of Thessaloniki, Greece

Correspondence: Georgios Tsoulfas, Aristotle University of Thessaloniki, 66 Tsimiski Street, Thessaloniki, Greece, Tel 306971895190, Fax 30-2310332022, Email tsoulfasg@gmail.com

Received: May 13, 2014 | Published: May 14, 2014

mapping technologies and translational bioinformatics, it has been possible to elucidate a number of genes and molecules that have the potential to act as biomarkers for the development of hepatic metastases as well as their response to the different therapeutic regiments. The emerging possibilities appear endless; however, there are several issues that remain. These include the high cost of this technology as well as the ethical and legal issues that can present significant challenges. Moreover, the original enthusiasm has to be tempered by the fact that from the hundreds of biomarkers that have been proposed, only very few, such as KRAS have actually been integrated to clinical practice as of yet. ${ }^{4}$

In order for the progress seen with the use of new surgical techniques and improved chemotherapeutic regiments in the management of hepatic metastases from CRC to continue, it is vital to understand and recognize the role of the organ and tumor microenvironment as well as the contribution of the patient's genomic identity. Once this happens, it will be possible to identify and implement therapeutic strategies that are patient targeted as they will be tailored to the patient's genomic signature. The results will be more focused. All of this underscores the need for proper and rigorous validation of data, so that it can be moved to the clinical stage. To achieve that it is essential that emphasis is given on the creation of data and tissue banks, so that the proper research can be conducted. If this recipe is followed, then the best is yet to come.

\section{Acknowledgements}

None.

\section{Funding}

None. 


\section{Conflicts of Interest}

The author declares that there is no conflicts of interest.

\section{References}

1. Tournigand $\mathrm{C}$, Andre $\mathrm{T}$, Achille E, et al. FOLFIRI followed by FOLFOX6 or the reverse sequence in advanced colorectal cancer: a randomized GERCOR study. J Clin Oncol. 2004;22(2):229-237.
2. Scheele J, Stangl R, Altendorf-Hofmann A. Hepatic metastases from colorectal carcinoma: impact of surgical resection on the natural history. Br J Surg. 1990;77(11):1241-1246.

3. Hernandez-Gea V, Toffanin S, Friedman SL, et al. Role of the microenvironment in the pathogenesis and treatment of hepatocellular carcinoma. Gastroenterology. 2013;144(3):512-527.

4. Braillon A. Hepatocellular carcinoma and gene profiling: back from perspective to reality. Hepatology. 2011;54(2):738-739. 